Case

Report

\title{
Three-Dimensional Printing Model of Anomalous Bronchi before Surgery
}

\author{
Tadashi Akiba, MD, PhD, Takuya Inagaki, MD, PhD, and Takeo Nakada, MD
}

\begin{abstract}
Lung surgeries in patients with bronchial variations have rarely been reported. Here, we describe the case of a patient along with lung cancer with variant anatomy of the right upper lobe bronchus. This variation was evaluated by three-dimensional multi-detector computed tomography angiography with bronchography and a three-dimensional printing model using rapid prototyping. The variant anterior segment bronchus $(\mathrm{S3})$ of the right upper lobe arising from the middle lobe bronchus was confirmed before surgery using the printing model, which helped to determine the extent of resection required and facilitated the understanding of the patient's anatomy during surgery. A thoracoscopic anterior segmentectomy and middle lobectomy were performed. The printing model was useful for detecting and evaluating the variant bronchi.
\end{abstract}

Keywords: rapid prototyping, bronchial anomaly, lung cancer surgery, thoracoscopic surgery

\section{Introduction}

Thoracoscopic segmentectomy of the lung is a procedure that is sometimes adopted for the surgical treatment of patients with lung cancer. However, the anatomy of the lungs is variable in different individuals. Various anatomical variants in bronchi and vessels may cause serious complications during these surgeries, especially during thoracoscopic surgery. Thus, a detailed preoperative evaluation of the patient's anatomy is essential for preventing these complications.

We evaluated a patient with an unusual bronchial variation using a three-dimensional (3D) printing model with rapid prototyping which enabled us to understand the bronchial variation in the patient before surgery. Here, we describe the case of a patient with lung cancer

Department of Surgery, Jikei University Kashiwa Hospital, Kashiwa, Chiba, Japan

Received: July 21, 2013; Accepted: August 7, 2013

Corresponding author: Tadashi Akiba, MD, PhD, FACS. Department of Surgery, Jikei University Kashiwa Hospital, 163-1 Kashiwashita, Kashiwa, Chiba 277-8567, Japan

Email: akiba@jikei.ac.jp

(C)2014 The Editorial Committee of Annals of Thoracic and Cardiovascular Surgery. All rights reserved. showing variant anatomy in the right lung who underwent a thoracoscopic anterior segmentectomy and a middle lobectomy using rapid prototyping, which facilitated the understanding of the patient's anatomy during the surgery.

\section{Method}

On the basis of data collected using contrast mediumenhanced multi-detector computed tomography (MDCT) scans and analyzed by the volume analyzer Synapse Vincent (Fujifilm Medical Co., Ltd., Tokyo, Japan), we were able to fabricate $3 \mathrm{D}$ mixed-color replicas of the pulmonary anatomy model using a printer (Connex, Stratasys Ltd., Eden Prairie, Minnesota, USA). In these models, the bronchi, pulmonary arteries, and veins appear in different colors because of differences in their density. We obtained institutional ethics committee approval before this research.

Patients scheduled for thoracic surgery at Jikei University Kashiwa Hospital are routinely examined using MDCT (Aquilion 64; Toshiba, Tokyo, Japan) after intravenous administration of iodinated contrast medium. Tailor-made virtual lungs are constructed from MDCT images of the pulmonary vessels and bronchi for each patient. ${ }^{1,2}$ 


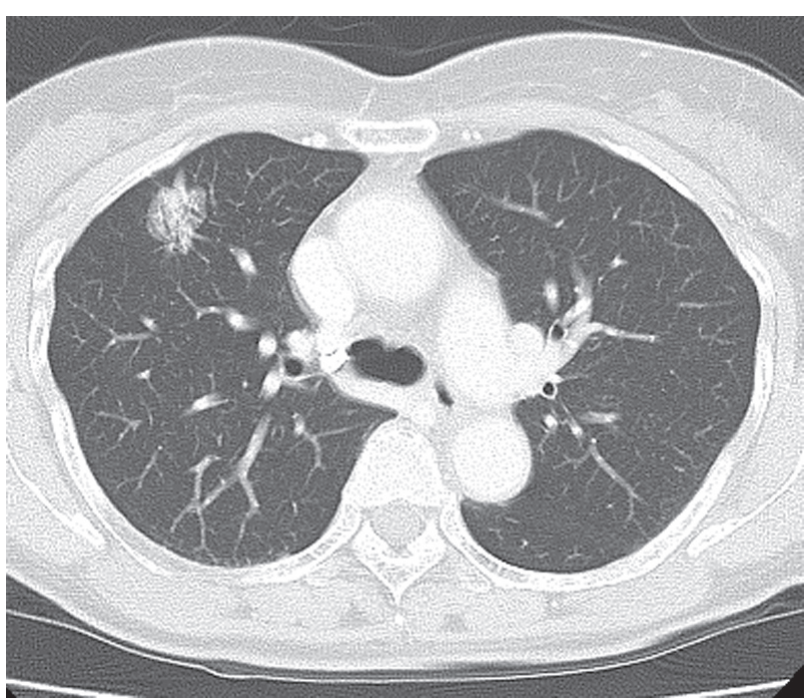

Fig. 1 Chest computed tomography (CT) showing groundglass opacity in the right lung.

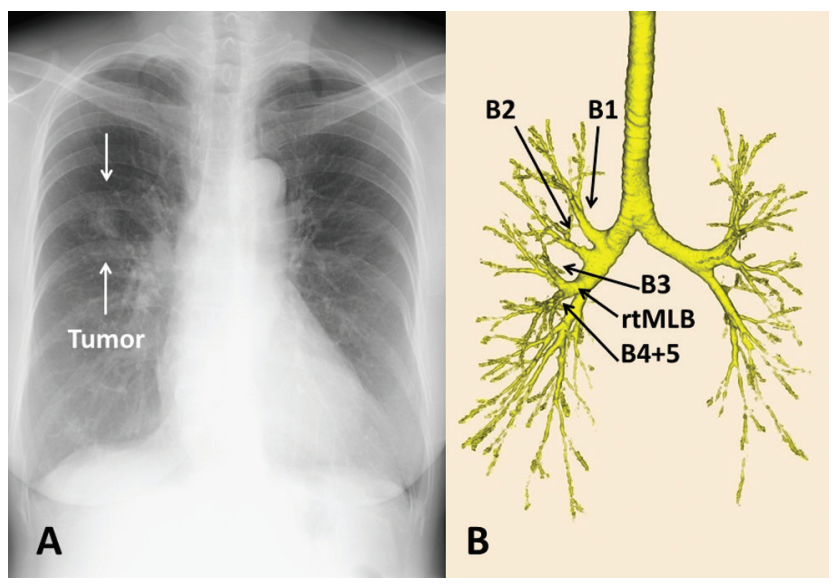

Fig. 2 (A) Chest roentgenogram showing an abnormal shadow in the right lung. (B) Chest computed tomography (CT) with bronchography showing variations in the following bronchi: the right apical (B1) and posterior bronchi (B2) of the right upper lobe directly originating from the right main bronchus; the right anterior bronchus (B3) originating from the right middle lobe bronchus (rtMLB). B4 + 5: the right lateral (B4) and medial bronchi (B5)of the right middle lobe $(\mathrm{B} 4+5)$.

\section{Case Report}

A 70-year-old woman was referred to our hospital for further evaluation of an abnormal shadow that was detected using a municipal roentgenogram examination. MDCT with contrast enhancement showed an abnormal shadow of ground-glass opacity that measured $2.6 \times 1.9 \mathrm{~cm}$ in the anterior segment $(\mathrm{S} 3)$ of the right

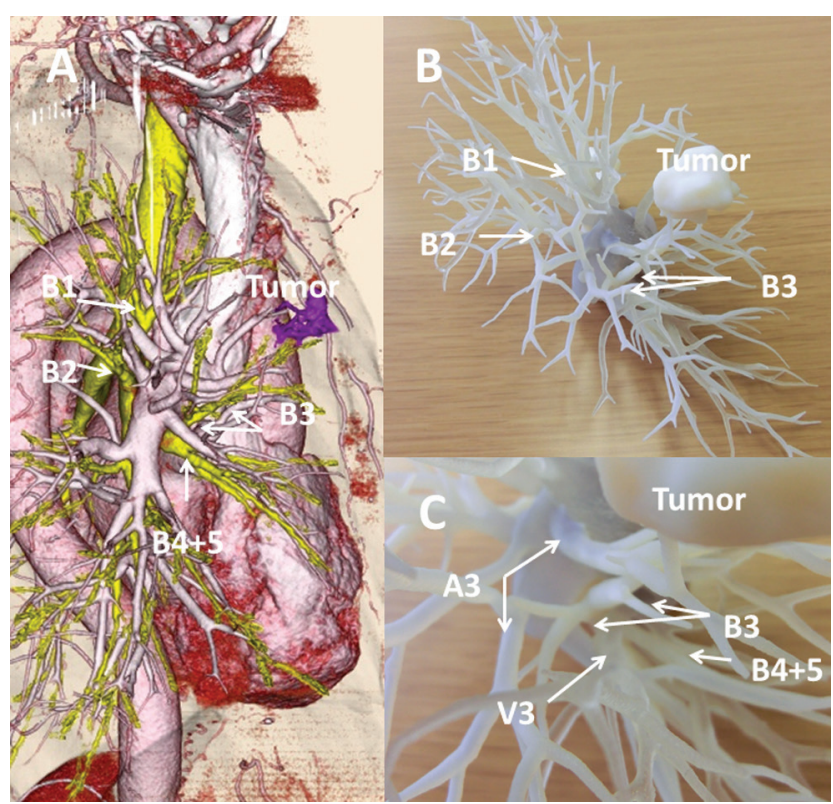

Fig. 3 (A) Chest computed tomography angiography (CTA) with bronchography. (B) Rapid prototyping model. (C) Close-up photography of the model. The anterior bronchus (B3), arteries (A3), and veins (V3) of the right upper lobe are observed. B1 and B2: the right apical and posterior bronchi of the right upper lobe. $\mathrm{B} 4+5$ : the right lateral (B4) and medial bronchi (B5) of the right middle lobe $(\mathrm{B} 4+5)$.

upper lobe, which was compatible with lung carcinoma (Figs. 1 and 2).

The 3D printing model revealed that the apical (B1) and posterior segment bronchi (B2) of the upper lobe directly arising from the right main bronchus that aerate the apical (S1) and posterior segments (S2), respectively, were anomalous (Figs. 2 and 3). The anterior segment bronchus (B3) that aerates S3 arose from the middle lobe bronchus instead of the right upper lobe bronchus. This variation was diagnosed to be a dislocation of the bronchus. In all these, the printing model provided good visualization of the variant bronchus and the related blood vessels.

The patient underwent a thoracoscopic anterior segmentectomy and a middle lobectomy that was combined with sampling of the regional lymph nodes. No interlobar fissures were observed between the upper and middle lobes, and a fissure was observed between the upper and the lower lobes. As for the bronchus, this procedure required only the division of the middle lobe bronchus. The vessels that were related to the middle lobe and S3 were divided. An interlobar plane was made using the 
staplers $3 \mathrm{~cm}$ away from the tumor.

The postoperative course was uneventful, and the patient was discharged on postoperative day 6. A pathological analysis of the resected specimen revealed adenocarcinoma, pT1aN0M0, stage IA.

\section{Discussion}

The use of thoracoscopic lobectomies for the treatment of patients with lung cancer has been increasing in many countries, and even thoracoscopic pulmonary segmentectomies have been gradually adopted in the field of thoracic surgery. ${ }^{1-3)}$ Anomalies of the pulmonary vessels are sometimes encountered, but tracheobronchial anomalies are not so frequent and bronchial variations have rarely been reported. ${ }^{4-6)}$

The most common developmental anomalies of the bronchial tree include accessory cardiac bronchus and tracheal bronchus. Displaced segmental bronchi, variants of tracheal bronchus, and bronchial agenesis constitute the minor bronchial anomalies. ${ }^{7}$ Tracheal bronchus may be in the form of accessory tracheal bronchus and aberrant tracheal bronchus. The latter arises from an abnormal position and aerates one or more segments of an upper lobe (usually the apical segment) in which case the corresponding normal segmental bronchus (or bronchi) is missing. ${ }^{7)}$

Suzuki, et al., reported 30 true tracheal bronchi among 9781 cases that were examined using MDCT. ${ }^{8)}$ Ohta, et al., reported a total of 85 tracheobronchial anomalies in 71 patients on the basis of a review of the bronchographies of 13222 cases. $^{9)}$ A total of $75 \%$ of the tracheobronchial anomalies were in the right upper lobe. A total of 59 displaced segmental bronchi were observed and 10 of them were from the middle lobe bronchus: one bronchus distributed to the $\mathrm{B} 2,8$ to the $\mathrm{B} 3$, and one to $\left.\mathrm{B} 2+3 .{ }^{9}\right)$ Furthermore, the variant bronchi in patients with lung cancer are even more rare. ${ }^{10,11)}$

We confirmed the bronchial anomaly of this case using MDCT angiography with bronchography and the $3 \mathrm{D}$ printing model. The present case exhibited that not only had the B1 and B2 bronchi displaced from the right main bronchus but also that the B3 was displaced from the middle lobe bronchus. These variant bronchi were detected prior to the thoracoscopic surgery. MDCT and the rapid prototyping allowed us to know the precise anatomy of the bronchi and the associated pulmonary vessels before the thoracoscopic surgery.

Bronchoscopic examinations are good methods for evaluating the segmental bronchi, but it is sometimes difficult to know the precise relationship between bronchi and the aerated pulmonary parenchyma. The MDCT and printing model showed not only the anomalous bronchial tree but also its aerated parenchyma. Because we knew the precise anatomy of the bronchi and the vessels, we were able to decide on the procedure involving the resection of S3 and the middle lobe by only dividing the middle lobe bronchi.

Rapid prototyping has proved to be a useful tool in maxillofacial surgery, reconstructive surgery, neurosurgery, orthopedics, and adult and pediatric cardiac surgery. ${ }^{12-15)}$ There have not been any studies describing the use of rapid prototyping in chest surgery or the use of the printing model for rapid prototyping of anomalous lungs. We believe that this method will have promising benefits in the near future in the field of thoracic surgery.

\section{Disclosure Statement}

We have no conflict of interest.

\section{References}

1) Akiba T, Morikawa T, Ohki T. Simulation of thoracoscopic surgery using 3-dimensional tailor-made virtual lung. J Thorac Cardiovasc Surg 2012; 143: 1232-4.

2) Akiba T, Morikawa T, Ohki T. Thoracoscopic lung segmentectomy simulated by a tailor-made virtual lung: computed bronchography and angiography. Thorac Cardiovasc Surg 2013; 61: 151-3.

3) Leshnower BG, Miller DL, Fernandez FG, et al. Videoassisted thoracoscopic surgery segmentectomy: a safe and effective procedure. Ann Thorac Surg 2010; 89: 1571-6.

4) Watabnabe $K$, Uese $K$, Higuchi $O$, et al. Threedimensional computed tomographic findings of bilateral tracheal bronchus. Pediatr Cardiol 2009; 30: 87-8.

5) Zhong YM, Jaffe RB, Zhu M, et al. CT assessment of tracheobronchial anomaly in left pulmonary artery sling. Pediatr Radiol 2010; 40: 1755-62.

6) Read R, St Cyr J, Marek J, et al. Bronchial anomaly of the right upper lobe. Ann Thorac Surg 1990; 50: 980-1.

7) Yildiz H, Ugurel S, Soylu K, et al. Accessory cardiac bronchus and tracheal bronchus anomalies: CT-bronchoscopy and CT-bronchography findings. Surg Radiol Anat 2006; 28: 646-9.

8) Suzuki M, Matsui O, Kawashima H, et al. Radioanatomical study of a true tracheal bronchus using 
multidetector computed tomography. Jpn J Radiol 2010; 28: 188-92.

9) Ohta S, Saito Y, Usuda K, et al. Tracheobronchial anomalies: report of 71 cases. J Jpn Society Bronchology $1985 ; \mathbf{8}$ : 122-30.

10) Akiba T, Marushima H, Takagi M, et al. Preoperative evaluation of a tracheal bronchus by three-dimensional 64-row multidetector-row computed tomography (MDCT) bronchography and angiography: report of a case. Surg Today 2008; 38: 841-3.

11) Sen S, Sentürk E, Pabuşçu E, et al. Upper lobectomy for lung cancer with true tracheal bronchus: a unique presentation. Arch Bronconeumol 2010; 46: 332-4. (in Spanish)

12) Sodian R, Schmauss D, Markert M, et al. Three- dimensional printing creates models for surgical planning of aortic valve replacement after previous coronary bypass grafting. Ann Thorac Surg 2008; 85: 2105-8.

13) Schmauss D, Gerber N, Sodian R. Three-dimensional printing of models for surgical planning in patients with primary cardiac tumors. J Thorac Cardiovasc Surg 2013; 145: 1407-8.

14) Ngan EM, Rebeyka IM, Ross DB, et al. The rapid prototyping of anatomic models in pulmonary atresia. J Thorac Cardiovasc Surg 2006; 132: 264-9.

15) Esses SJ, Berman P, Bloom AI, et al. Clinical applications of physical 3D models derived from MDCT data and created by rapid prototyping. AJR Am J Roentgenol 2011; 196: W683-8. 